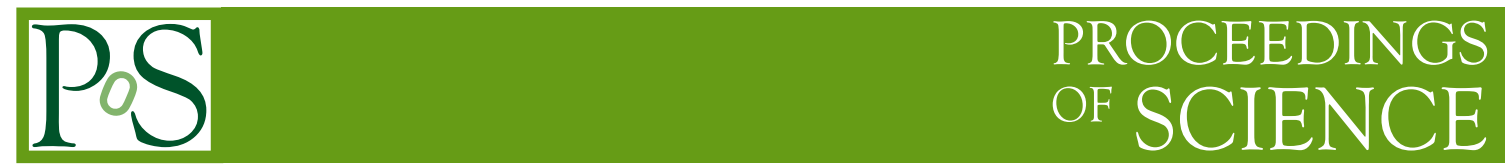

\title{
Long baseline neutrino oscillation experiments
}

\author{
André Rubbia* \\ ETH Zurich, 101 Rämistrasse, CH-8092 Zurich, Switzerland \\ E-mail: andre.rubbia@cern.ch
}

In the last decade, the study of flavor oscillations with neutrino long baseline experiments has been an extremely effective way to get information on the mass (up to an unknown scale) and the mixing between weak and mass neutrino eigenstates. In particular, accelerator driven experiments have determined precisely the so-called 2-3 sector and are beginning to tackle the 1-3 sector. Within a decade or two, there is hope to fully complete our understanding of the oscillation phenomenology between the three active neutrinos: the mass differences squared will have been very accurately determined and the neutrino mass hierarchy defined; the flavor mixing matrix, including its complex phase will also have been measured, possibly culminating with the discovery of (direct) CP-violation in the leptonic sector. The knowledge of the mass and flavor mixings of the leptons and quarks will shed light on the underlying properties of the fundamental fermions fields and is relevant for models of grand unified theories. In this paper, we briefly review the main achievements of this field and outline possible options for the future.

The 2011 Europhysics Conference on High Energy Physics-HEP 2011,

July 21-27, 2011

Grenoble, Rhone-Alpes France

${ }^{*}$ Speaker. 


\section{Introduction}

Just as classical optical or quantum mechanical interferometry has led to very high precision, long baseline neutrino oscillation experiments can be interpreted as "neutrino interferometry" studies, sensitive to otherwise practically unmeasurable effects. In such a setup, a neutrino is emitted at the source in a given weak eigenstate. This eigenstate is a linear combination of mass eigenstates, which form the diagonal basis for the evolution in vacuum space-time. At a distance from the source an interference pattern emerges, which leads to the observation of neutrino-energydependent flavor oscillations. Such a process is a powerful window to probe new physics, as any perturbation along the path will change the neutrino interference pattern at the far detector. For example, propagation through matter can lead to dramatic changes of the oscillation probabilities. In general, the oscillation pattern and deviations from its e.g. predicted energy dependence could reveal new unknown phenomena. In the last decade, the study of neutrino oscillations with long baseline experiments has been a very effective method to determine the neutrino mass spectrum (up to an unknown scale) and the mixing parameters. Within the next decade or two, there is hope to complete our understanding of the phenomenology of the oscillations between the three active neutrinos, possibly culminating with the discovery of (direct) CP-violation in the leptonic sector.

\section{The three neutrinos formalism and the corresponding oscillation probabilities}

In the three neutrino family scenario, the weak eigenstates $v_{\alpha}(\alpha=\mathrm{e}, \mu, \tau)$ are given as linear combinations of the mass eigenstates $v_{i}$ (of definite mass $\left.m_{i}, \mathrm{i}=1,2,3\right)$ via the PMNS[1] matrix $U$ as $v_{\alpha}=\sum_{i} U_{\alpha i} v_{i}$. The $3 \times 3$ unitary matrix $\mathrm{U}$ is parameterized by the three mixing angles $\theta_{12}, \theta_{13}$, $\theta_{23}$ and a complex phase $\delta_{C P}$. The probability of oscillation from flavor $\alpha$ to $\beta$ is given by $P\left(v_{\alpha} \rightarrow\right.$ $\left.v_{\beta}\right)=\delta_{\alpha \beta}-4 \sum_{i>j} \Re e\left(U_{\alpha i}^{*} U_{\beta i} U_{\alpha j} U_{\beta j}^{*}\right) \sin ^{2}\left(\hat{\Delta}_{i j}\right)+2 \sum_{i>j} \mathfrak{I} m\left(U_{\alpha i}^{*} U_{\beta i} U_{\alpha j} U_{\beta j}^{*}\right) \sin \left(2 \hat{\Delta}_{i j}\right)$ with $\hat{\Delta}_{i j} \equiv$ $\Delta m_{i j}^{2} L /(4 E)$ where $L$ is the distance between source and detector (the baseline), $E$ the neutrino energy and $\Delta m_{i j}^{2} \equiv m_{i}^{2}-m_{j}^{2}$. Experimental data indicate that $\Delta m_{21}^{2} \ll\left|\Delta m_{31}^{2}\right| \approx\left|\Delta m_{32}^{2}\right|$, whereby the 1-2(resp. 2-3) sectors describe solar (resp. atmospheric) oscillations.

\section{A decade of measurements of the $2-3$ sector}

Neglecting the 1-2 and 1-3 sectors (i.e. in two neutrino flavor), the 2-3 sector drives the muon disappearance with a simple expression $P\left(v_{\mu} \rightarrow v_{\mu}\right)=1-\sin ^{2} 2 \theta_{23} \sin ^{2}\left(\hat{\Delta}_{32}\right)$. A decade of precise measurements of the muon disappearance and the detection of one tau appearance candidate have confirmed the mechanism and allowed determining the $\Delta m_{32}^{2}$ and $\theta_{23}$ with high accuracy. The first long baseline neutrino oscillation experiment was K2K[2] in Japan. It had a baseline of $250 \mathrm{~km}$ and used Superkamiokande[3] (SK) as far detector with 22.5 kton fiducial mass. Its primary aim was to confirm the neutrino $v_{\mu} \rightarrow v_{\mu}$ disappearance effect observed in atmospheric neutrinos with a controlled man-made neutrino beam. The proton beam power was about $12 \mathrm{~kW}$ and $\mathrm{K} 2 \mathrm{~K}$ collected in total 107 fully contained events (which have no energy seen in the Outer Detector of SK) disfavoring the no-oscillation hypothesis at $4 \sigma$ C.L. The second experiment optimized for muon disappearance studies is MINOS [4] along the FNAL NUMI beam line, with a longer baseline than K2K of $730 \mathrm{~km}$ and a far detector target of 5.4 ktons. In 2006, MINOS confirmed the 
disappearance in the neutrino mode [5] with a 5.2 $\sigma$ C.L. and $\left|\Delta m^{2}\right|=2.74_{-0.22}^{+0.44} \times 10^{-3} \mathrm{eV}^{2}$ and $\sin ^{2} 2 \theta>0.87$ at $68 \%$ C.L. (here we have $\Delta m^{2} \approx \Delta m_{32}^{2}$ and $\sin ^{2} 2 \theta \approx \sin ^{2} 2 \theta_{23}$ ). In 2011, with an exposure of $7.25 \times 10^{20}$ protons-on-target (pot), MINOS provides the best measurement $\left|\Delta m^{2}\right|=$ $\left(2.32_{-0.08}^{+0.12}\right) \times 10^{-3} \mathrm{eV}^{2}$ and $\sin ^{2} 2 \theta>0.90$ at $90 \%$ C.L. [6]. Since MINOS is a magnetized detector, it can identify on an-event-by-event basis the charged-current muon antineutrino sample coming from the $7 \% \bar{v}_{\mu}$ contamination of the beam. It observed a number of antineutrino events consistent with $\left|\Delta \bar{m}^{2}\right|=2.32 \times 10^{-3} \mathrm{eV}^{2}$ and $\sin ^{2}(2 \bar{\theta})=1.0$ [7]. Using the more effective method of reversing the polarity of the horn focusing system to enhance the flux of antineutrinos, MINOS provided the first direct observation of muon antineutrino disappearance [8]. Based on $1.71 \times 10^{20}$ pot, the result was $\left|\Delta \bar{m}^{2}\right|=\left(3.36_{-0.40}^{+0.46}\right) \times 10^{-3} \mathrm{eV}^{2}$ and $\sin ^{2}(2 \bar{\theta})=0.86_{-0.12}^{+0.11} \pm 0.01$ (syst), in relative tension with the values measured for neutrinos. With an increased exposure in antineutrino mode of $2.95 \times 10^{20}$ pot, MINOS now reports preliminary results which are consistent with neutrinos, with best fit values $\left|\Delta \bar{m}^{2}\right|=\left(2.62_{-0.28}^{+0.31}(\right.$ stat $) \pm 0.09($ syst $\left.)\right) \times 10^{-3} \mathrm{eV}^{2}$ and $\sin ^{2}(2 \bar{\theta})=0.95_{-0.11}^{+0.10}($ stat $) \pm 0.01$ (syst $)$. OPERA[9] at the Gran Sasso Underground Laboratory was the first long baseline experiment in Europe. It has a hybrid lead plates and photographic emulsion film target, with a total mass of $1.25 \mathrm{kton}$. The CNGS beam is produced from $400 \mathrm{GeV}$ SPS protons. Based on an analyzed exposure of $1.89 \times 10^{19}$ pot, OPERA finds one tau candidate event over a background of $0.045 \pm 0.023$ (syst) [10]. Including the 2011 run, the CNGS has accumulated an exposure of $1.4 \times 10^{20}$ pot. OPERA is currently scanning the collected dataset for $v_{\mu} \rightarrow v_{\tau}$ candidates.

\section{The current race - quest for $\theta_{13}$}

The 1-3 sector drives the (subleading) appearance of electron neutrinos in an accelerator muon neutrino beam $v_{\mu} \rightarrow v_{e}$ with the probability $P\left(v_{\mu} \rightarrow v_{e}\right) \approx \sin ^{2} 2 \theta_{13} \sin ^{2} \theta_{23} \sin ^{2}\left(\hat{\Delta}_{31}\right)+$ higher order terms $f\left(\delta_{C P}, \theta_{12}\right)$. T2K [11] uses a conventional neutrino beam produced at J-PARC and directed $2.5^{\circ}$ off-axis to SK at a distance $L=295 \mathrm{~km}$. This configuration produces a narrowband $v_{\mu}$ beam, tuned at the first oscillation maximum $E_{v}=\left|\Delta m_{32}^{2}\right| L /(2 \pi) \simeq 0.6 \mathrm{GeV}$, reducing backgrounds from higher energy neutrino interactions. The T2K experiment observes indications of $v_{\mu} \rightarrow v_{e}$ appearance [12] in data accumulated with $1.43 \times 10^{20}$ protons on target. Six events pass all selection criteria at the far detector. In a three-flavor neutrino oscillation scenario with $\left|\Delta m_{32}^{2}\right|=2.4 \times 10^{-3} \mathrm{eV}^{2}, \sin ^{2} 2 \theta_{23}=1$ and $\sin ^{2} 2 \theta_{13}=0$, the expected number of such events is $1.5 \pm 0.3$ (syst.). Under this hypothesis, the probability to observe six or more candidate events is $7 \times 10^{-3}$, equivalent to $2.5 \sigma$ significance. At $90 \%$ C.L., the data are consistent with $0.03(0.04)<$ $\sin ^{2} 2 \theta_{13}<0.28(0.34)$ for $\delta_{\mathrm{CP}}=0$ and normal (inverted) hierarchy. T2K data taking suddenly stops after the March 11 earthquake and is getting ready to resume continuous running by February 2012. NOvA [13] is a long-baseline neutrino oscillation experiment which aims at finding the appearance of electron neutrinos in both the NuMI neutrino and antineutrino beam modes. It is comprised of a near detector located on-site at FNAL and a 14 kton far detector located $810 \mathrm{~km}$ from the source in northern Minnesota. Both detectors are positioned 14 mrad off the beam axis to observe a narrow range of neutrino energies peaked at $2.2 \mathrm{GeV}$. NOvA has a basic element composed of a PVC extrusion filled with liquid scintillator. The scintillation light is readout by avalanche photodiodes (APD). Construction of the NOvA experiment has begun and the detector should be fully assembled by fall 2013. The projected sensitivities of both T2K and NOvA for 
$\theta_{13}$ are very similar. In the case of NOvA, the longer baseline will allow probing difference in $v_{e}$ appearance between neutrino and antineutrino modes, induced by matter effects. The ability to resolve the mass hierarchy as normal at the $95 \% \mathrm{CL}$ is possible for about $50 \%$ of the $\mathrm{CP}$ values for $\sin ^{2} 2\left(2 \theta_{13}\right)>0.07$.

\section{Future steps - the neutrino mass hierarchy and looking for $\mathrm{CP}$ violation}

Beyond the present round of experiments, a set of ideas have emerged which consider optimized scenarios at long baselines to settle the neutrino mass hierarchy in a conclusive way and/or to unveil CP-violation in the leptonic sector. Including the higher order terms and also the possibility for matter effects, the $v_{\mu} \rightarrow v_{e}$ (resp. $\left(\bar{v}_{\mu} \rightarrow \bar{v}_{e}\right)$ ) probabilities can be written as[14]: $\left.P\left(v_{\mu} \rightarrow v_{e}\right)\left(\operatorname{resp} . P\left(\bar{v}_{\mu} \rightarrow \bar{v}_{e}\right)\right)\right)=P_{0} \mp P_{\sin \delta}+P_{\cos \delta}+P_{3}$ where

$$
\begin{aligned}
P_{0} & =\sin ^{2} \theta_{23} \frac{\sin ^{2} 2 \theta_{13}}{(\hat{A}-1)^{2}} \sin ^{2}\left((\hat{A}-1) \hat{\Delta}_{31}\right) \\
P_{\sin \delta} & =\alpha \frac{\sin \delta_{C P} \cos \theta_{13} \sin 2 \theta_{12} \sin 2 \theta_{13} \sin 2 \theta_{23}}{\hat{A}(1-\hat{A})} \sin \left(\hat{\Delta}_{31}\right) \sin \left(\hat{A} \hat{\Delta}_{31}\right) \sin \left((1-\hat{A}) \hat{\Delta}_{31}\right) \\
P_{\cos \delta} & =\alpha \frac{\cos \delta_{C P} \cos \theta_{13} \sin 2 \theta_{12} \sin 2 \theta_{13} \sin 2 \theta_{23}}{\hat{A}(1-\hat{A})} \cos \left(\hat{\Delta}_{31}\right) \sin \left(\hat{A} \hat{\Delta}_{31}\right) \sin \left((1-\hat{A}) \hat{\Delta}_{31}\right) \\
P_{3} & =\alpha^{2} \frac{\cos ^{2} \theta_{23} \sin ^{2} 2 \theta_{12}}{\hat{A}^{2}} \sin ^{2}\left(\hat{A} \hat{\Delta}_{31}\right)
\end{aligned}
$$

with $\Delta m_{21}^{2}=\alpha \Delta m_{31}^{2}$ and $\hat{A}= \pm A / \Delta m_{31}^{2}= \pm 2 V E_{V} / \Delta m_{31}^{2}$.

There are two main modes of investigations: (a) the first method is to measure the $\mathrm{CP}$ asymmetry by comparing the appearance rates of neutrinos and antineutrinos $[v / \bar{v} \mathrm{CP}$ asymmetry]; it requires both beam polarities with similar statistics which is more challenging for antineutrinos due to lower flux and cross-sections; it can be performed in a narrow band beam and experimentally requires a good control of systematic errors between neutrino and antineutrino runs; (b) the second method is to look for $v_{e}\left(\bar{v}_{e}\right)$ appearance and to measure its energy spectrum shape in a wide-band beam (WBB) at a given baseline $L$ [CP with WBB]. The fit of the CP-phase is basically determined by the peak positions and height of the 1st and 2nd maximum and minimum. This method is sensitive to all values of $\delta_{C P}$ and allows determining the value of $\delta_{C P}$ using neutrino only. However, degeneracies require the neutrino mass hierarchy to be determined beforehand. In a wide band beam and if the baseline is sufficiently large, the hierarchy and the $C P$-phase can be determined simultaneously and unambiguously with 50\%-50\% neutrino and antineutrino modes. Experimentally this measurement requires good energy resolution and low background systematics.

Table 1 lists options and some assumed parameters for future long baseline neutrino experiments presently considered in Europe, Japan and USA. In Japan two options along the JPARC neutrino beam built for $\mathrm{T} 2 \mathrm{~K}$ and upgraded to $1.66 \mathrm{MW}$ are debated. The first option considers a 560 kton water Cerenkov detector for proton decay and neutrino astrophysics (Hyperkamiokande [15]) located near the present location of SK at Kamioka with a baseline of $295 \mathrm{~km}$. The second option envisions a giant liquid argon detector on the Okinoshima island [16] at a distance of $658 \mathrm{~km}$. A LoI for Hyperkamiokande has been recently presented [17]. In USA the LBNE project [18] has received "mission need" from the Department of Energy and is presently 
investigating two detector technologies (Water Cerenkov and Liquid Argon) to be located at the Homestake mine at a distance of $1300 \mathrm{~km}$ from FNAL. The European options are being studied within the LAGUNA/LAGUNA-LBNO design study [19], funded by the European Commission and composed of institutions from EU, Japan, Russia and USA. After several years of detailed comparison between seven potential sites and three detector technologies, the study is presently focusing on two main complementary options: (a) a giant water Cerenkov detector located at the Fréjus site with a baseline of $130 \mathrm{~km}$ from CERN; (b) a giant liquid Argon detector located at the farthest possible location from CERN situated at a baseline of $2300 \mathrm{~km}$ at the Pyhäsalmi mine in Finland. The LAGUNA-LBNO consortium intends to submit by summer 2012 an EoI to the CERN Strategy group updating the European Roadmap for Particle Physics. The CERN-Fréjus baseline offers a great synergy with a potential beta-beam from CERN [20] while the CERN-Pyhäsalmi baseline is already optimized for a potential future neutrino factory [21]. Both European options therefore show very good prospects for the very long term.

Table 1: Past, present and possibly future long baseline neutrino experiments, ordered by baseline. SK stands for SuperKamiokande. The indicated proton driver powers are design parameters except for KEKPS, CNGS and NUMI which correspond to achieved values, indicated by $(*)$.

\begin{tabular}{|c|c|c|c|c|}
\hline $\begin{array}{l}\text { Beam / } \\
\text { future facility }\end{array}$ & Proton driver & Baseline & $\begin{array}{l}\text { Detector / } \\
\text { fid. mass }\end{array}$ & $\begin{array}{c}\text { Primary } \\
\text { physics goal } \\
\text { with beam }\end{array}$ \\
\hline \multicolumn{5}{|c|}{ Past and present } \\
\hline KEK K2K & PS $12 \mathrm{GeV}, 0.012 \mathrm{MW}(*)$ & $250 \mathrm{~km}$ & SK 22.5 kton & $v_{\mu} \rightarrow v_{\mu}$ \\
\hline FNAL NUMI & MI $120 \mathrm{GeV}, 0.3 \mathrm{MW}(*)$ & $735 \mathrm{~km}$ & MINOS 5.4 kton & $v_{\mu} \rightarrow v_{\mu}$ \\
\hline CERN CNGS & SPS $400 \mathrm{GeV}, 0.3 \mathrm{MW}(*)$ & $732 \mathrm{~km}$ & OPERA 1.25 kton & $v_{\mu} \rightarrow v_{\tau}$ \\
\hline & & & ICARUS 0.6 kton & $v_{\mu} \rightarrow v_{\tau}$ \\
\hline J-PARC T2K & MR $30 \mathrm{GeV}, 0.75 \mathrm{MW}$ & $295 \mathrm{~km}$ & SK 22.5 kton & $v_{\mu} \rightarrow v_{e}$ \\
\hline FNAL NUMI+ & $\mathrm{MI}+120 \mathrm{GeV}, 0.75 \mathrm{MW}$ & $810 \mathrm{~km}$ & NOvA 14 kton & $v_{\mu} \rightarrow v_{e}$ \\
\hline \multicolumn{5}{|c|}{ Future options } \\
\hline $\begin{array}{c}\text { CERN LAGUNA- } \\
\text { Fréjus }\end{array}$ & HP-SPL $5 \mathrm{GeV}, 4 \mathrm{MW}$ & $130 \mathrm{~km}$ & WC 440 kton & $v / \bar{v}$ CP asymm \\
\hline JPARC-Kamioka & $\mathrm{MR}+, 1.66 \mathrm{MW}$ & $295 \mathrm{~km}$ & WC 560 kton & $v / \bar{v}$ CP asymm \\
\hline JPARC-Okinoshima & $\mathrm{MR}+, 1.66 \mathrm{MW}$ & $658 \mathrm{~km}$ & LAr 100 kton & CP with WBB \\
\hline FNAL-LBNE & $\begin{array}{c}\text { MI+, } 0.75 \mathrm{MW} \\
(2.3 \mathrm{MW} \text { ProjectX) }\end{array}$ & $1300 \mathrm{~km}$ & $\begin{array}{l}\text { WC } 200 \text { kton } \\
\text { or LAr } 34 \text { kton }\end{array}$ & $\begin{array}{l}\text { CP+hierarchy } \\
\text { with WBB }\end{array}$ \\
\hline $\begin{array}{l}\text { CERN LAGUNA- } \\
\text { Pyhäsalmi }\end{array}$ & $\begin{array}{c}\text { SPS+, } 0.7 \mathrm{MW} \\
\text { (HP-PS } 50 \mathrm{GeV}, 2 \mathrm{MW})\end{array}$ & $2300 \mathrm{~km}$ & LAr 100 kton & $\begin{array}{l}\mathrm{CP}+\text { hierarchy } \\
\text { with WBB }\end{array}$ \\
\hline
\end{tabular}

\section{Conclusions}

The past, present and possibly future long baseline neutrino experiments are summarized in Table 1. After a decade of very successful experimentation that has lead to the precise measurement of the 2-3 sector and the beginning of the understanding of the 1-3 sector, there continued to be 
a world-wide interest for next generation long-baseline based on the conventional neutrino beam technology as a further step beyond T2K/NOvA, with longer baselines to address CP-violation and mass hierarchy. The physics case is strongly reinforced by recent evidence for $\sin ^{2} 2 \theta_{13}>$ 0.01 . As a community, we should aim at realizing at least two complementary future projects (but many technical and financial challenges lie ahead). Worldwide coordination is surely necessary. In Europe, the LAGUNA consortium will consider an incremental (staged) approach towards CPviolation, with a first step aimed at a definite and guaranteed determination of the neutrino mass hierarchy.

\section{References}

[1] Z. Maki, M. Nakagawa and S. Sakata, Prog. Theor. Phys. 28, 870 (1962). B. Pontecorvo, Sov. Phys. JETP 26, 984 (1968) [Zh. Eksp. Teor. Fiz. 53, 1717 (1967)].

[2] E. Aliu et al. [K2K Collaboration], Phys. Rev. Lett. 94, 081802 (2005) [hep-ex/0411038].

[3] Y. Fukuda, T. Hayakawa, E. Ichihara, M. Ishitsuka, Y. Itow, T. Kajita, J. Kameda and K. Kaneyuki et al., Nucl. Instrum. Meth. A 501, 418 (2003).

[4] E. Ables et al. [MINOS Collaboration], FERMILAB-PROPOSAL-0875.

[5] D. G. Michael et al. [MINOS Collaboration], Phys. Rev. Lett. 97, 191801 (2006) [hep-ex/0607088].

[6] P. Adamson et al. [The MINOS Collaboration], Phys. Rev. Lett. 106, 181801 (2011) [arXiv:1103.0340 [hep-ex]].

[7] P. Adamson et al. [MINOS Collaboration], Phys. Rev. D 84, 071103 (2011) [arXiv:1108.1509 [hep-ex]].

[8] P. Adamson et al. [MINOS Collaboration], Phys. Rev. Lett. 107, 021801 (2011) [arXiv:1104.0344 [hep-ex]].

[9] M. Guler et al. [OPERA Collaboration], CERN-SPSC-2000-028.

[10] N. Agafonova et al. [OPERA Collaboration], Phys. Lett. B 691, 138 (2010) [arXiv:1006.1623 [hep-ex]].

[11] K. Abe et al. [T2K Collaboration], Nucl. Instrum. Meth. A 659, 106 (2011) [arXiv:1106.1238 [Unknown]].

[12] K. Abe et al. [T2K Collaboration], Phys. Rev. Lett. 107, 041801 (2011) [arXiv:1106.2822 [hep-ex]].

[13] D. S. Ayres et al. [NOvA Collaboration], FERMILAB-DESIGN-2007-01.

[14] M. Freund, Phys. Rev. D 64, 053003 (2001) [hep-ph/0103300].

[15] K. Nakamura, Front. Phys. 35, 359 (2000).

[16] A. Badertscher et al., arXiv:0804.2111 [hep-ph].

[17] K. Abe, T. Abe, H. Aihara, Y. Fukuda, Y. Hayato, K. Huang, A. K. Ichikawa and M. Ikeda et al., arXiv:1109.3262 [hep-ex].

[18] T. Akiri et al. [LBNE Collaboration], arXiv:1110.6249 [hep-ex].

[19] A. Rubbia [LAGUNA Collaboration], Acta Phys. Polon. B 41 (2010) 1727.

[20] M. Benedikt, A. Bechtold, F. Borgnolutti, E. Bouquerel, L. Bozyk, J. Bruer, A. Chance and P. Delahaye et al., Eur. Phys. J. A 47, 24 (2011).

[21] S. Choubey et al. [The IDS-NF Collaboration], arXiv:1112.2853 [Unknown]. 